\title{
Béatrice Bakhouche, Calcidius. Commentaire au Timée de Platon
}

\section{Gretchen Reydams-Schils}

\section{(2) OpenEdition}

1 Journals

Édition électronique

URL : https://journals.openedition.org/philosant/716

DOI : 10.4000/philosant.716

ISSN : 2648-2789

Éditeur

Éditions Vrin

\section{Édition imprimée}

Date de publication : 1 novembre 2016

Pagination : 234-237

ISBN : 978-2-7574-1472-9

ISSN : 1634-4561

\section{Référence électronique}

Gretchen Reydams-Schils, « Béatrice Bakhouche, Calcidius. Commentaire au Timée de Platon », Philosophie antique [En ligne], 16 | 2016, mis en ligne le 01 novembre 2018, consulté le 04 décembre 2022. URL : http://journals.openedition.org/philosant/716 ; DOI : https://doi.org/10.4000/philosant. 716

\section{(c) $($ ) $(9)$}

Creative Commons - Attribution - Pas d'Utilisation Commerciale - Pas de Modification 4.0 International - CC BY-NC-ND 4.0

https://creativecommons.org/licenses/by-nc-nd/4.0/ 
stoïcien chez Stobée sous l'angle de la metis, qu'elle rapproche de la phronesis que les stoïciens héritent de Platon. Parler de la metis du sage stoïcien se justifie par le fait que le mensonge est considéré par les stoïciens comme parfois légitime, utile et convenable, et par leur intérêt pour le personnage d'Ulysse. L'auteur fait alors un lien entre l'éloge de la polutropia comme forme de sagesse et l'habileté dialectique du sage qui peut aller jusqu'à lui donner une allure quasi-sophistique. On complétera cet article par celui d'Ilaria Ramelli (« Ierocle neostoico in Stobeo : і каөп́коvт e l'evoluzione dell'etica stoica », p. 537-576), qui analyse l'évolution morale stoïcienne à partir de la présence de Hiéroclès. Ce même thème est repris par Emanuele Vimercati («Stobeo sul saggio stoico », p. 577-614). À l’opposé du caractère tranché de la dichotomie sage-insensé dans le stoïcisme hellénistique, Stobée insiste sur un perfectionnement moral qui transige avec la rigidité de la doctrine de ces derniers. Cette conclusion valide l'idée que l'Anthologie obéit avant tout à une visée pédagogique. Selon Julie Giovacchini («Techniques de discours et techniques de vérité chez Stobée : remarques sur le Peri parrhesias (III 13) », p. 615-631), c'est précisément son projet pédagogique qui fait de Stobée, non pas un compilateur laborieux mais un auteur de manuel philosophique à part entière, la dimension protreptique de l'ouvrage comportant évidemment une dimension réflexive et critique dans les choix des excerpta retenus. Se fondant à la fois sur le livre III, chapitre 13 de Stobée et sur le De adulatore de Plutarque, l'auteur montre que tous deux élèvent la parrhesia à un rang philosophique, entre protreptique et elenchos socratique. Le recours à Plutarque aide ainsi à reconnaitre dans l'Anthologie une œuvre à vocation philosophique.

Le seul regret que nous aurions à exprimer porte sur la densité du propos et la présentation un peu «labyrinthique » de ce livre-somme, qui ne peut s'adresser qu’à un public expert. Si les études stobéennes en bénéficieront certainement, il vise plutôt un public de philologues, la critique dominant largement les arguments et les analyses conceptuelles. Malgré l'aridité qu'une telle entreprise devait sans doute impliquer, c'est bien une nouvelle étape dans la compréhension de l'œuvre de Stobée qui vient d'être franchie avec succès.

\section{Olivier D'Jeranian Université Paris 1 Panthéon-Sorbonne}

Béatrice Bakhouche, Calcidius. Commentaire au Timée de Platon, édition critique et traduction française (avec la collab. de Luc Brisson), 2 tomes, Paris, Vrin, 2011 (Histoire des Doctrines de l'Antiquité Classique, 42), 934 p., ISBN 978-2-71162264-1.

On peut difficilement contester l'importance de la traduction du Timée (17a-53c) par un certain Calcidius, accompagnée d'un commentaire d'une section plus courte (31c-53c), datant du IV e siècle de notre ère. Comme le note Béatrice Bakhouche (p. 58), ce texte est, avec le Commentaire au Songe de Scipion de Macrobe, les Noces de Mercure et Philologie de Martianus Capella et la Consolation de Philosophie de Boèce, un des quatre « maîtres-livres » qui ont assuré la transmission de l'héritage philosophique au Moyen-Âge. De la fin de l'Antiquité jusqu'au XII ${ }^{\mathrm{e}}$ siècle, Platon se résumait presque uniquement à ce texte. C'est un texte difficile, œuvre d'un auteur autrement inconnu, écrit dans un latin compliqué souvent calqué sur le grec, et dont le contenu couvre de nombreux domaines, allant, dans la première partie du commentaire, des mathématiques à la musique et à l'astronomie. Béatrice Bakhouche avait déjà traduit et commenté pour sa thèse de doctorat la première partie du commentaire. Ce nouveau travail magistral 
permettra d'élargir et d'enrichir les recherches sur ce texte, ouvrant la voie à une meilleure compréhension du commentaire dans son rapport avec la traduction.

Cette nouvelle édition complète les conjectures avancées par Waszink lui-même depuis la publication de sa propre édition en $1962^{1}$. Elle représente un progrès considérable dans le choix des figures et dans l'étude du rapport de ces figures avec le texte dans la première partie du commentaire. B. Bakhouche (p. 103) recense environ trente différences avec le texte de Waszink. Les notes relatives à la traduction soulignent pour la première fois les divergences entre la traduction et l'original. Ces divergences sont essentielles à la compréhension des liens existant entre la traduction et le commentaire ainsi qu'à la méthodologie employée par le commentateur.

À titre d'exemple, les notes de B. Bakhouche sur la traduction du passage qui traite des connaissances de l'âme du monde (Timée, 37a2-c5) permettent de voir que déjà dans sa traduction Calcidius a simplifié et interprété ce passage pour ses lecteurs. Au lieu de rendre les tournures compliquées de ce passage, Calcidius établit dans la traduction ellemême un lien entre, d'une part, le Même, l'Être indivisible et la connaissance, et d'autre part, l'Autre, l'Être divisible et l'opinion. Dans sa traduction, l'âme du monde voit « les causes de tout ce qui se passe » et peut prévoir l'avenir - ce dernier élément n'étant pas présent dans le Timée lui-même.

Voici un deuxième exemple du type de recherches facilitées par le travail de B. Bakhouche. Comme l'a noté A. Galonnier ${ }^{2}$, quand Calcidius discute la question très controversée de la relation entre le monde et le temps (une question cruciale dans toute la tradition des discussions du Timée), il élimine des ambiguités très nettes que

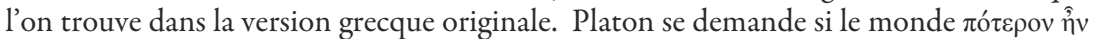

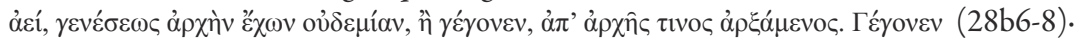

En grec, arche peut indiquer un commencement dans le temps aussi bien qu'un rapport causal (tout comme le latin initium qu'utilise Calcidius), et gegonen peut signifier « est devenu » ou « est en état de devenir ». Dans la traduction de Calcidius, en revanche, nous lisons :

Item mundus fueritne semper citra exordium temporis an sit originem sortitus ex tempore, considerandum - factus est ...

Il souligne ici nettement la dimension temporelle en ajoutant deux fois tempus, un ajout qui détermine alors le sens de factus est, qui de plus en latin évoque la question de savoir par qui ou par quoi le monde a été fait. Cependant, comme l'a remarqué Christina Hoenig ${ }^{3}$, cette traduction ne contredit pas la position du commentateur qui se range dans le camp de ceux qui donnent une interprétation « allégorique » du passage de Platon, en arguant que le langage du temps est plus accessible au commun des hommes qu'un rapport purement causal entre le monde et le dieu. Autrement dit, Calcidius commence par simplifier le texte de Platon en éliminant certaines ambiguités avant d'en découvrir le sens caché à son lecteur.

Revenons au travail de B. Bakhouche. L'introduction au texte, accompagnée d'une deuxième introduction spécialement destinée à la traduction, est une véritable mine

1. Jan Hendrik Waszink, Timaeus a Calcidio translatus commentarioque instructus, Leiden-London, 1962 (Corpus Platonicum Medii Aevi, Plato Latinus 4).

2. Alain Galonnier, « Cosmogenèse et chronocentrisme chez Calcidius », Philosophie Antique, 9 (2009), p. 189-207.

3. Christina Hoenig, « Timaeus Latinus : Calcidius and the creation of the universe », Rhizomata, 2 (2014), p. 80-110. 
d'or. Des tableaux indiquent la relation entre les parties du Timée et le commentaire (p. 27-28) ou présentent une comparaison entre la traduction de Cicéron et celle de Calcidius (p. 105-124). B. Bakhouche fait aussi une nouvelle mise au point des rapports entre Calcidius et les premières générations potentiellement influencées par l'auteur, notamment des parallèles que l'on trouve entre Calcidius et Favonius Eulogius (p. 47-55). Comme le remarque B. Bakhouche elle-même (p. 47), l'hypothèse de Waszink que Calcidius, n'étant qu'un auteur « médiocre », ne pouvait pas être à l'origine des innovations relatives aux termes techniques du langage philosophique latin, n'est pas tenable. Mais dès qu'on admet que l'influence a pu aussi aller dans l'autre direction, c'est-à-dire de Calcidius aux autres auteurs, il est possible de rouvrir le débat concernant la date à laquelle le commentaire a été écrit. Il ne reste alors que la question des clausules métriques, qui ne sont pas un instrument très fiable pour déterminer la date d'une œuvre. Même la conclusion d'E. Mensching, reprise par B. Bakhouche (p. 34), sur la base de son analyse de l'Épître qui précède l'œuvre, ne permet de postuler qu'une marge assez large entre Arnobe (qui est mort vers 330) et Favonius Eulogius. De fait, la conclusion que le texte a dû être écrit « autour des années 400 » ne peut s'imposer avec force. Contre Waszink, qui tendait à repousser le texte au plus tard possible (même aux premières décennies du $v^{e}$ siècle), peut-être le moment est-il venu de reconsidérer la date de l'œuvre et de la situer davantage vers la moitié du IV .

Les notes du deuxième volume dépassent largement les analyses de Waszink, aussi bien que celles de Den Boeft et de Van Winden ${ }^{4}$, et permettent de situer Calcidius plus clairement dans la tradition philosophique. Mais puisque Calcidius a une affinité prononcée avec un platonisme qui est largement antérieur à Plotin (le moyen platonisme), comme l'affirme B. Bakhouche (p. 41), cette affinité conforte la thèse d'une datation moins tardive.

Le travail de B. Bakhouche, dans son analyse des sources (p. 34-41 et les Notes) nous permet de relativiser considérablement l'importance de Porphyre. Dans la réédition du texte en 1975, Waszink (p. CLXXXVII) allait jusqu'à postuler que le commentaire sur le Timée de Porphyre était la source principale de Calcidius. Sodano, dans sa collection de fragments du commentaire au Timée de Porphyre, a inclus des passages entiers du commentaire de Calcidius. ${ }^{5}$. C'est une approche qui n'est pas sans risques. Non seulement il y a un nombre important de divergences entre ce que nous savons des positions de Porphyre et celles de Calcidius, mais on ne peut pas non plus traiter la question des sources sans avoir une meilleure idée de la « voix » du commentateur lui-même et de ses prises de position que l'on retrouve dans l'ensemble du texte. Calcidius est loin d'être un simple compilateur des idées d'autrui.

Une présupposition mériterait d'être remise en question plus que ne le fait B. Bakhouche, à savoir l'identité chrétienne de Calcidius. Bien que B. Bakhouche se montre plus prudente que C. Moreschini ${ }^{6}$ dans l'attribution au commentateur de traits chrétiens, il reste néanmoins dans son travail des passages dans lesquels la présupposition

4. Jan den Boeft, Calcidius On Fate : His Doctrine and Sources, Leiden 1970 (Philosophia Antiqua, 23) et Calcidius On Demons (Commentarius 127-136), Leiden, 1977 (Philosophia Antiqua, 18) ; Jacobus C. M. Van Winden, Calcidius on Matter; His Doctrine and Sources : A Chapter in the History of Platonism, Leiden 1959 (Philosophia Antiqua, 9).

5. Angelo R. Sodano, Porphyrii in Platonis Timaeum Commentariorum Fragmenta, Naples, 1964.

6. Claudio Moreschini, Calcidio, Commentario al Timeo di Platone, Milan, 2003 (Il pensiero occidentale). 
d'une identité chrétienne influence trop la traduction. Par exemple, la traduction de cette phrase du chap. 126 : ad humanae conservationis rerumque mortalium gratiam par «... le salut de l'homme et la résurrection des mortels » introduit la notion spécifique de résurrection là où Calcidius embrasse une notion plus large, qui concerne la conservation des choses mortelles jusqu'à y inclure l'univers dans son intégralité. Dans le Timée lui-même Platon garantit la survie de l'univers par la volonté du Démiurge (41b4-5). La formulation de Calcidius permet aussi bien une interprétation chrétienne qu'une interprétation qui ressortirait plutôt à la tradition philosophique.

Dans la transmission du texte, la traduction était souvent séparée du commentaire, et entre la traduction et le commentaire de Calcidius lui-même s'est insérée une tradition riche et complexe de gloses. Cette approche de l'œuvre de Calcidius, dont d'ailleurs le nom souvent disparaissait derrière celui de Platon, ne permettait pas de mettre en valeur la continuité et la structure du texte. Mais une autre approche, plus récente, qui traite le texte comme une simple collection des sources antérieures pose le même problème. C'est grâce au travail novateur de B. Bakhouche que nous pouvons enfin mieux comprendre la méthodologie du commentateur qu'était Calcidius.

Gretchen Reydams-Schils University of Notre Dame 